\title{
Evaluation of safety and efficacy of NexGen - an ultrathin strut and hybrid cell design cobalt-chromium bare metal stent implanted in a real life patient population - the Polish NexGen Registry
}

\author{
Krzysztof Milewski ${ }^{1}$, Paweł Gąsior ${ }^{1}$, Stefan Samborski ${ }^{1}$, Piotr P. Buszman ${ }^{1}$, Aleksandra Błachut ${ }^{1}$, \\ Adam Wojtaszczyk ${ }^{1}$, Adam Młodziankowski ${ }^{1}$, Artur Mendyk ${ }^{1}$, Marek Król' ${ }^{1}$, Wojciech Wojakowski ${ }^{2}$, \\ Paweł E. Buszman ${ }^{1}$
}

${ }^{1}$ Center for Cardiovascular Research and Development, American Heart of Poland SA, Katowice, Poland

${ }^{2}$ Third Division of Cardiology, Medical University of Silesia, Katowice, Poland

\begin{abstract}
A bstract
Introduction: Despite the dominance of drug-eluting stents in modern interventional cardiology, there is still a niche for bare metal stents.

Aim: The aim of the Polish NexGen registry was to evaluate the safety and efficacy of a new generation cobalt-chromium NexGen stent in a real life patient population.

Material and methods: A prospective multi-center registry was conducted in five clinical sites of American Heart of Poland. Three hundred and eighty-three patients who underwent percutaneous coronary intervention $(\mathrm{PCl})$ with NexGen stent implantation were included. Clinical follow-up was performed at 1, 6 and 12 months. Additionally, a group of 42 randomly selected patients underwent control angiography at 6 months (10.96\% of study population). The primary endpoint was occurrence of target vessel revascularization (TVR) at 6-month follow-up. Angiographic endpoints included rates of binary restenosis and late lumen loss at 6-month follow-up based on QCA analysis. Multivessel disease was present in more than $70 \%$ of patients, and $52.4 \%$ of lesions were complex. The main indications for angiography were non-ST elevation acute coronary syndromes (54.8\%) and ST elevation myocardial infarction (34.99\%).

Results: At 6-month follow-up 47 (12.7\%) patients reached the primary endpoint of TVR. The composite of major acute cardiac event rates at 30-day and 6- and 12-month follow-up was $6.01 \%(n=23), 18.5 \%(n=69)$ and $25.21 \%(n=92)$ respectively. Control angiography performed after 6 months showed in-stent late loss of $0.66 \pm 0.71 \mathrm{~mm}$ and a binary restenosis rate of $16.7 \%$.

Conclusions: Our study showed that PCI with the NexGen stent is safe and effective at 6- and 12-month follow-up. Angiographic results showed a satisfactory restenosis rate and low late lumen loss.
\end{abstract}

Key words: stent, cobalt-chromium, ultrathin struts, hybrid cell design, all-comers patient population.

\section{Introduction}

For the last few years, new generation drug-eluting stents (DES) have become the treatment of choice for the vast majority of patients undergoing percutaneous coronary interventions $(\mathrm{PCl})$. Drug-eluting stents have proved to be more efficacious than bare metal stents (BMS), especially in reduction of the restenosis rate and the need for repeated revascularization [1]. Despite the dominance of DES in modern interventional cardiology, there is still a niche for bare metal stents. Bare metal stents may be considered in the situation of triple antiplatelet therapy using novel oral anticoagulants (NOAC) or double antiplatelet therapy with acetylsalicylic acid and clopidogrel in patients with high bleeding risk (HAS-BLED score $\geq 3$ ). In such clinical scenarios there are still no data from randomized trials that could recommend new generation DES over BMS [2], and the choice of stent needs to be made on an individual basis. Therefore, it is necessary to further improve stent design by introducing new materials or material modifications, decreasing strut thickness or by changing mechanical parameters of stents. New generation bare metal stents are characterized by ultra thin struts - a feature that improves their flexibility and deliverability. It also reduces vessel wall injury during 
implantation, which leads to faster endothelialization and a lower rate of restenosis [3, 4]. Another important feature that may decrease vessel wall injury is an asymmetrical pattern of stent opening, which puts less stress on stent edges. The new generation NexGen (Meril Life Science Pvt. Ltd, Vapi, Gujarat, India) cobalt-chromium bare metal stent combines all these features in a single device.

\section{Aim}

The aim of the Polish NexGen Registry was to determine the safety and efficacy of the NexGen stent in a "real world" patient population

\section{Material and methods}

\section{Device description}

The balloon expandable NexGen bare metal stent (Meril Life Sciences Pvt, Ltd., Vapi, Gujarat, India) is based on an $L 605$ cobalt-chromium platform, which incorporates ultra-thin struts $(65 \mu \mathrm{m})$ with a unique hybrid cell design comprising a mix of open and closed cells in order to provide optimal radial strength (recoil < $3 \%$ ) and uncompromised side branch access (open cells in the middle) without worsening flexibility (Figure 1). The design of the balloon allows balloon-related edge injury to be minimized due to abrupt shoulders and minimal overhang (once crimped). It also allows morphology-mediated expansion from the middle (center) to the edges during deployment (Figure 1).

\section{Study design}

The NexGen Registry is a prospective multi-center registry conducted in 5 clinical sites located in Poland. The study was performed with the approval of the local Ethical Committee. Informed, written consent was obtained from all study participants. The registry includes patients hospitalized between January 2010 and June 2011 who underwent $\mathrm{PCl}$ with the NexGen stent and who were reachable by telephone contact at 30-day follow-up. Clinical follow-up (FU) was performed by telephone contact at three time points: 30 days, 6 months and 12 months after discharge. During telephone contacts information was gathered on any major adverse cardiac or cerebrovascular events as well as on angina status and cardiovascular drug use. There were no limitations to the number of treated vessels, lesions, lesion length and diameter. In the case of multiple lesions, all lesions were treated with the NexGen stent. The exclusion criteria were as follows: contraindications to dual antiplatelet therapy (DAPT), cardiogenic shock, prolonged resuscitation before the procedure and use of stents other than NexGen. Initially, a group of 50 randomly selected patients was scheduled for control coronary angiography in order to evaluate stent efficacy based on binary restenosis and late lumen loss at 6-month follow-up. Eventually, 42 of them consented and underwent elective coronary angiography $(10.96 \%$ of the study population). Percutaneous coronary intervention was performed using standard techniques. Pharmacological treatments recommended by the European Society of Cardiology were introduced before and after intervention. All patients received both aspirin (75 mg once daily) and clopidogrel (75 mg once daily) for 30 days in the case of stable coronary artery disease and 12 months in the case of acute coronary syndromes, followed by aspirin alone thereafter. Predilatation was not obligatory and depended on the operator's preferences.

\section{Definitions and end points}

Hemodynamically significant lesions were defined as $\geq 50 \%$ diameter stenosis in the left main (LM) and proximal segment of the left anterior descending (LAD) coronary arteries or $\geq 70 \%$ diameter stenosis in other segments. Angiographic success was defined as residual stenosis $<20 \%$ in the presence of TIMI flow 3 after

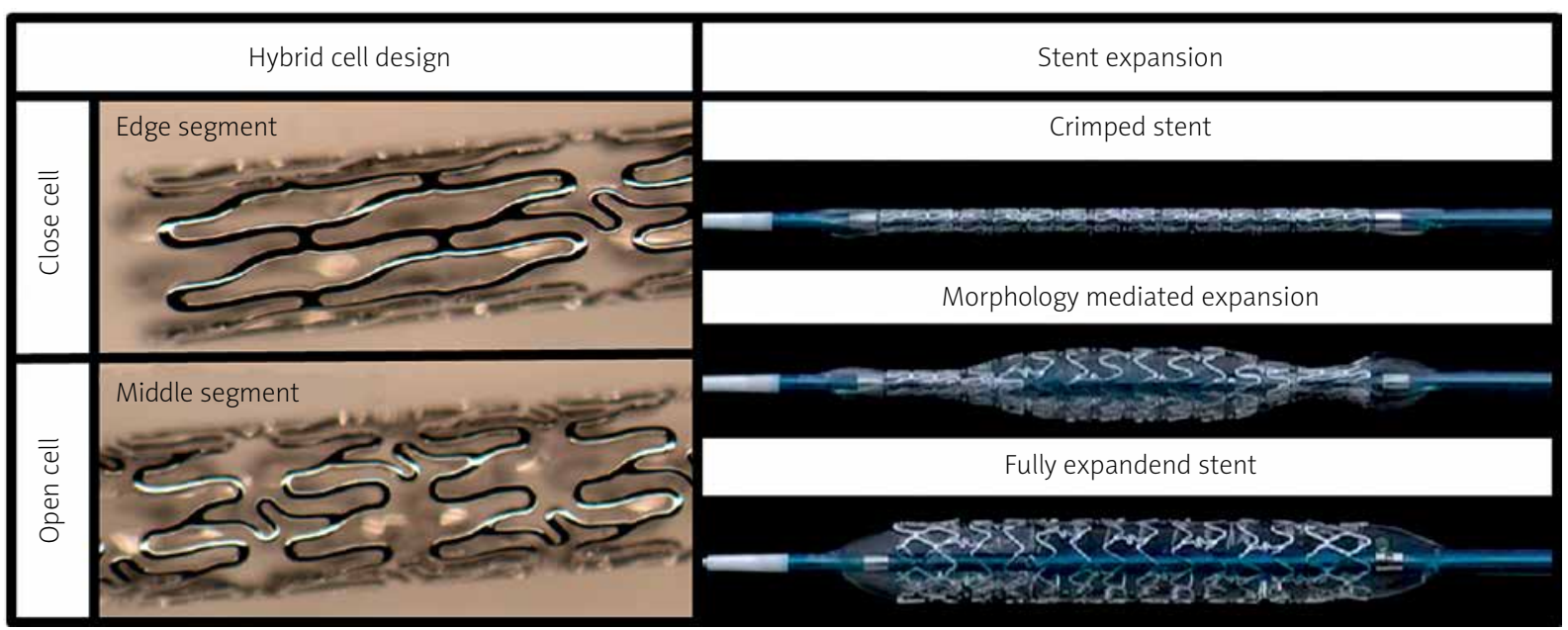

Figure 1. Hybrid cell design and morphology mediated expansion of NexGen stent 
studied device placement [5]. Procedural success was achieved with a satisfactory angiographic outcome in the absence of peri-procedural major adverse cardiac events (MACE) defined as a composite of cardiac death, myocardial infarction and clinically indicated target lesion revascularization according to the definitions of the Academic Research Consortium (ARC) [6]. Successful delivery and deployment of NexGen with withdrawal of the stent delivery system together with confirmation of angiographic success was considered as device success.

The primary efficacy endpoint was the occurrence of target vessel revascularization (TVR) defined as a repeat intervention (surgical or percutaneous) to treat luminal stenosis within the epicardial artery previously treated with the studied device at 6-month FU.

Secondary endpoints included clinically indicated target lesion revascularizations (TLR), MACE, all-cause death, cardiac death and stent thrombosis evaluated at 30-day, 6- and 12-month follow-up. Additionally, device success and procedural success were assessed. By protocol, all deaths were considered cardiac unless a non-cardiac cause could be clearly established by either clinical assessment or pathological study. Stent thrombosis was classified based on timing of occurrence after stent implantation and was considered as acute ( 0 to $24 \mathrm{~h}$ ), sub-acute (> $24 \mathrm{~h}$ to 30 days), or late (> 30 days). Stent thrombosis was defined as certain if it had been confirmed during coronary angiography or during post-mortem examination. Probable stent thrombosis was diagnosed when a heart attack occurred in the area supplied by the previous stented artery and was not confirmed by coronary angiography. Angiographic endpoints included rates of binary restenosis and late lumen loss at 6-month follow-up based on quantitative coronary angiography (QCA) analysis.

\section{Angiographic analysis}

Quantitative coronary angiography measurements were performed independently by two experienced interventional cardiologists. Two contralateral projections were chosen for stent assessment. Angiograms of stented segments were analyzed in views showing most severe stenosis at the following time-points: before intervention, after intervention and at 6-month follow-up. Measurements were made based on calibration of a contrast-filled catheter and automatic contour detection of the treated segment using an automated edge detection algorithm (MEDIS, Cardiovascular angiography, the Netherlands). Reference vessel diameter (RVD) was calculated as the arithmetic mean of vessel diameter in segments proximal and distal to the target lesion. Percent diameter stenosis (\%DS) was defined as the difference between RVD and minimal lumen diameter (MLD) divided by the reference diameter multiplied by 100 . Late loss (LL) was defined as the difference be- tween MLD in the angiogram obtained after stent implantation and at the follow-up. Binary restenosis was defined as stenosis of $50 \%$ or greater of the MLD in the target lesion at angiographic follow-up. Acute gain was defined as the change in the MLD from baseline to the final procedural angiogram.

\section{Statistical analysis}

All clinical and angiographic continuous variables with normal distribution are expressed as mean \pm SD. Continuous variables without normal distribution are expressed as median with interquartile range. Normal distribution of variables was verified by the Kolmogorov-Smirnov and Lilliefors test for normality.

\section{Results}

\section{Demographic characteristics}

The analysis consists of baseline clinical data (383 patients) and consecutive follow-up data collected at 1 month (383 patients), 6 months (373 patients, $2.61 \%$ lost to follow-up) and 12 months (365 patients, $4.7 \%$ lost to follow-up). Of 50 patients randomly scheduled for control angiography at 6-month FU, 42 consented and underwent elective procedure. The majority of patients were male, and mean age was 66.6 years (Table I). The incidence of diabetes mellitus was $30.29 \%$. Current smok-

Table I. Baseline clinical characteristics

\begin{tabular}{lcc} 
Parameter & Number & $\begin{array}{c}\text { Percentage or standard } \\
\text { deviation }\end{array}$ \\
\hline Age & 66.6 & SD $=10.22$ \\
\hline Male & 257 & $67.1 \%$ \\
\hline Female & 126 & $32.9 \%$ \\
\hline Diabetes mellitus & 116 & $30.29 \%$ \\
\hline Hypertension & 311 & $81.41 \%$ \\
\hline Dyslipidemia & 192 & $50.13 \%$ \\
\hline Current smoker & 87 & $22.72 \%$ \\
\hline History of MI & 109 & $28.46 \%$ \\
\hline History of PCI & 93 & $24.28 \%$ \\
\hline History of CABG & 12 & $3.13 \%$ \\
\hline SA & 39 & $10.18 \%$ \\
\hline UA & 127 & $33.16 \%$ \\
\hline NSTEMI & 83 & $21.67 \%$ \\
\hline STEMI & 134 & $34.99 \%$ \\
\hline LVEF (\%) & 50.66 & SD $=10.2$ \\
\hline
\end{tabular}

MI - myocardial infarction, $P C l$ - percutaneous coronary intervention, CABG coronary artery bypass graft, SA - stable angina, UA - unstable angina, NSTEMI - non-ST segment elevation myocardial infarction, STEMI - ST segment elevation myocardial infarction, LVEF - left ventricular ejection fraction. 
ers constituted $22.72 \%$ of patients. History of myocardial infarction (MI) and prior revascularization procedures occurred in a substantial number of screened patients, respectively in $28.46 \%$ and $27.41 \%$. The main indication for angiography was non-ST elevation acute coronary syndromes (NSTE-ACS) (54.8\%). ST elevation myocardial infarction (STEMI) occurred in $34.99 \%$ and stable angina (SA) in $10.18 \%$ of patients enrolled in the study.

Table II. Baseline angiographic characteristics

\begin{tabular}{|c|c|c|}
\hline Variable & Number & $\begin{array}{c}\text { Percentage or } \\
\text { interquartile range }\end{array}$ \\
\hline 1-vessel CAD & 105 & $27.42 \%$ \\
\hline 2-vessel CAD & 135 & $35.25 \%$ \\
\hline 3-vessel CAD & 143 & $37.44 \%$ \\
\hline \multicolumn{3}{|l|}{ Lesions distribution: } \\
\hline LM & 24 & $6.27 \%$ \\
\hline LAD & 247 & $64.49 \%$ \\
\hline$C x$ & 230 & $60.05 \%$ \\
\hline RCA & 303 & $79.11 \%$ \\
\hline SVG & 6 & $1.57 \%$ \\
\hline LIMA & 1 & $0.26 \%$ \\
\hline Ostial lesion & 17 & $4.51 \%$ \\
\hline Bifurcations & 29 & $7.71 \%$ \\
\hline Thrombus & 123 & $32.62 \%$ \\
\hline \multicolumn{3}{|c|}{ ACC/AHA lesion type based on core lab (42 patients): } \\
\hline Type A & 7 & $16.7 \%$ \\
\hline Type B1 & 13 & $31.0 \%$ \\
\hline Type B2 & 16 & $38.1 \%$ \\
\hline Type C & 6 & $14.3 \%$ \\
\hline \multicolumn{3}{|c|}{ Number of stents per lesion: } \\
\hline 1 & 276 & $72.44 \%$ \\
\hline 2 & 94 & $24.67 \%$ \\
\hline 3 & 8 & $2.1 \%$ \\
\hline 4 & 2 & $0.52 \%$ \\
\hline 5 & 1 & $0.26 \%$ \\
\hline $\begin{array}{l}\text { Average stent length per } \\
\text { lesion }[\mathrm{mm}]\end{array}$ & 29.0 & IQR: 19.0-40.0 \\
\hline $\begin{array}{l}\text { Mean stent diameter } \\
{[\mathrm{mm}]}\end{array}$ & 3.0 & IQR: $2.75-3.50$ \\
\hline $\begin{array}{l}\text { Mean inflation pressure } \\
{[\text { atm] }}\end{array}$ & 16.0 & IQR: 14.0-18.0 \\
\hline Direct stenting & 175 & $46.54 \%$ \\
\hline
\end{tabular}

\section{Clinical and angiographic outcomes}

During hospitalization one patient experienced myocardial infarction due to acute in-stent thrombosis and was successfully treated with balloon angioplasty (Table III). Four (1.04\%) patients died. At 6-month FU 47 (12.7\%) patients reached the primary endpoint of TVR. The composite of MACE rates at 30-day, 6- and 12-month FU was $6.01 \%, 18.5 \%$ and $25.21 \%$ respectively. The rates of $\mathrm{MI}$ after 30 days, 6 and 12 months were 1.83\%, 3.49\% and $4.38 \%$ respectively (Table IV). There was one acute stent thrombosis and 1 case of late stent thrombosis during 12-month follow-up (Table III).

Baseline QCA analysis showed mean percent diameter stenosis of $80.93 \pm 19.28 \%$ (Table V). Post procedure in-stent \%DS was significantly reduced to $14.42 \%$ $\pm 7.21 \%$ with acute gain of $2.06 \pm 0.60 \mathrm{~mm}$. Control angiography performed after 6 months showed in-stent late loss of $0.66 \pm 0.71 \mathrm{~mm}$ and a binary restenosis rate of $16.67 \%$ ( 7 cases; 3 of them were focal and another 4 affected the entire stent).

\section{Discussion}

In this study we tested the new generation balloon expandable NexGen bare metal stent, which is based on an ultra-thin cobalt-chromium platform and utilizes a hybrid cell design allowing for morphology mediated expansion from the middle to the edges. Such construction should allow for fast arterial healing and favorable

Table III. Rate of stent thrombosis during hospitalization and follow-up

\begin{tabular}{lcc} 
Stent thrombosis & Number & Percentage \\
\hline Acute ST (hospitalization) & 1 & 0.26 \\
\hline ST at 6-month follow-up & 1 & $0.27(0.26)$ \\
\hline ST at 12-month follow-up & 2 & $0.54(0.52)$
\end{tabular}

ST - stent thrombosis. 
procedural results. Thin struts assure low blood flow perturbance, and theoretically faster endothelialization and reduction of in-stent restenosis [3, 7]. In experimental settings it has been shown that NexGen stents significantly reduced neointimal thickness 28 days after implantation $(p=0.004)$ as compared to bare metal stent with strut thickness of $105 \mu \mathrm{m}$ [8]. Similarly, thin struts allow the production of stents with an extremely low profile that helps in device deliverability and flexibility. In addition, asymmetrical stent opening may reduce the incidence of margin dissection.

In our study, the majority of included patients fall into the category of the all-comers registry representative of everyday clinical practice of treatment of acute coronary

Table IV. Rate of composite MACE and individual components of MACE and stroke at 30-day, 6- and 12-month follow-up

\begin{tabular}{|c|c|c|}
\hline Variable & Number & Percentage \\
\hline \multicolumn{3}{|l|}{ Thirty-day follow-up $(n=383)$ : } \\
\hline MACE & 23 & 6.01 \\
\hline TVR & 10 & 2.61 \\
\hline TLR & 10 & 2.60 \\
\hline Subacute stent thrombosis & 1 & 0.26 \\
\hline Myocardial infarction & 7 & 1.83 \\
\hline Stroke & 0 & 0.00 \\
\hline All cause death & 9 & 2.35 \\
\hline \multicolumn{3}{|l|}{ 6-month follow-up $(n=373)$ : } \\
\hline MACE & 69 & 18.50 \\
\hline TVR & 47 & 12.60 \\
\hline TLR & 39 & 10.46 \\
\hline Stent thrombosis & 1 & 0.27 \\
\hline Myocardial infarction & 13 & 3.49 \\
\hline Stroke & 4 & 1.07 \\
\hline All cause death & 14 & 3.75 \\
\hline \multicolumn{3}{|l|}{ 12-month follow-up $(n=365)$ : } \\
\hline MACE & 92 & 25.21 \\
\hline TVR & 65 & 17.81 \\
\hline TLR & 50 & 13.70 \\
\hline Stent thrombosis & 2 & 0.54 \\
\hline Myocardial infarction & 16 & 4.38 \\
\hline Stroke & 5 & 1.37 \\
\hline All cause death & 19 & 5.21 \\
\hline
\end{tabular}

MACE - major adverse cardiac events, TVR - target vessel revascularization, $T L R$-target lesion revascularization. syndromes in Poland. Indeed, $89.82 \%$ of patients in the study presented with acute coronary syndromes. In addition, a substantial population consisted of elderly patients, having coexisting diseases which might require early discontinuation of dual antiplatelet therapy, or patients who are likely to be non-compliant. Importantly, one third of patients had 3-vessel disease, total stent length per lesion was $29.0 \mathrm{~mm}$, and most of the lesions were classified as complex (B2 and C based on the AHA/ ACC classification). Despite this challenging anatomy, the device success rate was $99.78 \%$ and procedural success was achieved in $98.7 \%$. In addition, direct stenting was used in almost half of cases (46.54\%). These data suggest good stent flexibility and deliverability. The percentage of margin dissection in the whole treated population was very low $(0.7 \%)$ and could be explained by the asymmetrical pattern of stent implantation.

Acute stent thrombosis confirmed by coronary angiography occurred in $1(0.22 \%)$ case and was successfully treated with balloon angioplasty. In-hospital mortality was low at $1.04 \%$. For comparison, in the large analysis performed by Badheka et al. in-hospital mortality in the BMS group was 1.4\% [9].

At 1-month follow-up TVR was $2.6 \%$ and no certain case of stent thrombosis occurred. Mortality at this time

Table V. Quantitative coronary artery analysis

\begin{tabular}{lc} 
Parameter & Value \\
\hline Before implantation: & $0.57 \pm 0.63$ \\
\hline MLD [mm] & $2.96 \pm 0.77$ \\
\hline RD [mm] & $80.93 \pm 19.28$ \\
\hline \%DS & $92.74 \pm 8.41$ \\
\hline \%AS & \\
\hline MLD [mm] & $2.60 \pm 0.57$ \\
\hline RD [mm] & $3.03 \pm 0.56$ \\
\hline \%DS & $14.42 \pm 7.21$ \\
\hline \%AS & $26.30 \pm 11.86$ \\
\hline Acute gain [mm] & $2.06 \pm 0.60$ \\
\hline 6-month follow-up: & $1.98 \pm 0.94$ \\
\hline MLD [mm] & $2.90 \pm 0.67$ \\
\hline RD [mm] & $33.09 \pm 24.77$ \\
\hline \%DS & $49.24 \pm 24.84$ \\
\hline \%AS & $0.66 \pm 0.71$ \\
\hline Late loss [mm] & $16.67 \%(n=7)$ \\
\hline Binary restenosis &
\end{tabular}

$M L D$ - minimal lumen diameter, RD - reference diameter, \%DS - \% diameter stenosis, \%AS - \% area stenosis. 
point was $2.35 \%$. At 6-month follow-up TVR as the primary endpoint of the study occurred in $12.6 \%$ of cases. Although a few earlier reports of BMS use showed a lower rate of TVR, the total stent length used in their studies was significantly shorter than that in our study (between $15.6 \mathrm{~mm}$ and $20.3 \mathrm{~mm}$ in 7 BMS reports vs. $31.5 \mathrm{~mm}$ in our study) [10-16]. In addition, although in clinical studies including the SOLSTICE registry the authors tested their stents in complex real world settings, all included patients had only one stent per lesion (number of lesions = 292 and number of stents $=293$ ) [12]. This is in contrast to our study, in which $27.55 \%$ of patients had more than one stent implanted ( 2 stents were implanted in $24.67 \%$ of patients and more than 2 in $2.88 \%$ of patients), and in our opinion it is closer to the real world patient population, explaining any differences in the results. During the whole study period there was only one case of definite late stent thrombosis $(0.27 \%)$, and death occurred in $3.75 \%$ of patients.

Importantly, the QCA analysis revealed relatively low late lumen loss of $0.66 \pm 0.71 \mathrm{~mm}$ and a favorable restenosis rate of $16.67 \%$, especially in terms of the use of BMS in a complex patient population.

All these data confirm the safety of NexGen stents and efficacy at the level found in other BMS. A recent study by Urban et al. demonstrated higher safety and efficacy of third generation polymer-free Biolimus-eluting stents when used with 1-month DAPT in a selected population of patients at high risk of bleeding when compared to BMS [17]. However, the stent used in the mentioned study was $72 \%$ thicker $(112 \mu \mathrm{m})$ than NexGen, which may have impacted the results. Additionally, the latest ESC guidelines on myocardial revascularization still recommend DAPT administration for at least 1 month after BMS and 6 months after new generation DES implantation in patients with stable coronary artery disease [18]. Therefore the conducted study suggests that in a selected group of patients including older patients with high risk of bleeding, BMS may still be a valuable option to treat coronary artery disease. Based on published data, also other clinical and economic situations may be justified to use these stents. In a prospective collection of data from 31 centers in Europe and Asia, the authors aimed to identify the main reason for implantation of BMS rather than DES in 744 consecutive PCls. They found eight indications for using BMS including large vessel diameter (32.4\%); ST-segment elevation myocardial infarction (17.7\%); reimbursement/regulatory/other reasons (9.4\%); advanced age (12.4\%); concomitant oral anticoagulant treatment (11.3\%); increased bleeding risk, cancer, or anemia (9.5\%); planned noncardiac surgery within the next year (5.5\%); and anticipated poor DAPT compliance (1.7\%) [19].

Although the NexGen Registry is rather a short-term assessment of safety and efficacy, it is widely accepted for bare metal stent technology evaluation. The proposed method of clinical evaluation in the study had its disadvantages. Telephone contact was satisfactory in routine cases but not sufficient especially in case of death. The patient's family often did not provide full information, and determining the cause of death was often impossible. In such cases, death was always regarded as cardiac, which could result in overestimation. Another problem that the investigators encountered was hospitalization in units other than randomizing. Then MACE was often difficult to classify, especially in cases with a lack of medical documentation. Investigators always accepted the least favorable version for the study. These facts could lead to false clinical results. Importantly, at the time of the registry the penetration of DES in Poland was approximately $40 \%$ due to the reimbursement practices, but nowadays it is almost $80 \%$, so the data provide the safety profile of BMS but have less value in the contemporary practice of widespread DES use. The potential advantage of the NexGen stent is its asymmetrical expansion, which theoretically reduces edge dissection. Unfortunately, in our study we were using only angiographic evaluation of arteries, which is limited by relatively low resolution and does not enable one to precisely evaluate edge dissection as OCT could precisely do. This study was a single arm, open-label registry; therefore one of the major limitations of this study was a lack of randomized comparison to another type of therapy. Finally, although two independent interventional cardiologists performed QCA analysis, this should be optimally done in an external and independent core laboratory.

\section{Conclusions}

Our study showed that the NexGen stent is safe and effective BMS technology. The presented data should be interpreted in the context of the progress of BMS design in a similar manner as in DES. Clearly the technology of stent construction, especially reduction of strut thickness, should affect the outcomes, especially late lumen loss and restenosis rate. Overall, interim data from the Polish NexGen registry showed satisfactory outcomes in terms of clinical events. Angiographic results were also encouraging to create DES based on the NexGen platform for further improvements of endovascular procedure quality.

\section{Acknowledgments}

This study was financially supported by Meril Life Sciences Pvt. Ltd.

\section{Conflict of interest}

The authors declare no conflict of interest.

\section{References}

1. Kastrati A, Mehilli J, Pache J, et al. Analysis of 14 trials comparing sirolimus-eluting stents with bare-metal stents. N Engl J Med 2007; 356: 1030-9. 
2. Kohl P, Windecker S, Alfonso F, et al. 2014 ESC/EACTS guidelines on myocardial revascularization: the task force on myocardial revascularization of European Society of Cardiology (ESC) and European Association for Cardio-Thoracic Surgery (EACTS). Eur Heart J 2014; 35: 2541-619.

3. Kastrati A, Mehilli J, Dirshinger J, et al. Intracoronary stenting and angiographic results: strut thickness effect on restenosis outcome (ISAR-STEREO) trial. Circulation 2001; 103: 2816-21.

4. Pache J, Kastrati A, Mehilli J, et al. Intracoronary stenting and angiographic results: strut thickness effect on restenosis outcome (ISAR-STEREO-2) trial. J Am Coll Cardiol 2003; 41: 1283-8.

5. Levine GN, Bates ER, Blankenship JC, American College of Cardiology Foundation; American Heart Association Task Force on Practice Guidelines; Society for Cardiovascular Angiography and Interventions. 2011 ACCF/AHA/SCAI Guideline for Percutaneous Coronary Intervention. J Am Coll Cardiol 2011; 58: e44-122.

6. Cutlip DE, Windecker S, Mehran R, et al. Clinical end points in coronary stent trials. Circulation 2007; 115: 2344-51.

7. Simon C, Palmaz JC, Sprague EA. Influence of topography on endothelialization of stents: clues for new designes. J Lon Term Eff Med Implants 2000; 10: 143-51.

8. Sheiban I. Evolution of NexGen. Angioplasty Summit TCTAP 2011.

9. Badheka AO, Arora S, Panaich SS, et al. Impact on in-hospital outcomes with drug-eluting stents versus bare-metal stents. Am J Cardiol 2014; 114: 1629-37.

10. Vermeersch P, Appelman Y, Horstkotte D, et al. Safety and efficacy of the cobalt chromium PRO-Kinetic coronary stent system: results of the MULTIBENE study. Cardiovasc Revasc Med 2012; 13: 316-20.

11. Strehblow C, Gyöngyösi M, Zenker G, et al. Small vessel stenting with cobalt-chromium stents (Arthos Pico) in a real-world setting. Coron Artery Dis 2007; 18: 305-11.

12. Suttorp MJ, Stella PR, Dens J, et al. Ultra-thin strut cobalt chromium bare metal stent usage in a complex real-world setting. (SOLSTICE registry). Neth Heart J 2015; 23: 124-9.

13. Bocksch W, Pomar F, Dziarmaga M, et al. Clinical safety and efficacy of the CoroFlex Blue cobalt-chromium stent system: results of the real-world CoroFlex Blue registry. Catheter Cardiovasc Interv 2010; 75: 78-85.

14. Giordano A, Polimeno M, Corcione N, et al. Synergy between direct coronary stenting technique and use of the novel thin strut cobalt chromium Skylor stent: the Mace in follow up patients treated with Skylor stent (MILES study). Curr Cardiol Rev 2012; 8: 6-13.

15. Kereiakes DJ, Cox DA, Hermiller JB, et al. Usefulness of a cobalt chromium coronary stent alloy. Am J Cardiol 2003; 92: 463-6.

16. Brambilla N, Morici N, Bedogni F, et al. Thin strut chrome-cobalt stent implantation for treatment of de novo lesions in small coronary vessels: results of the RISICO Italian registry (Registro Italiano Mini Vision nei piccolo Vasi) utilizing the mini vision coronary stent platform. J Cardiovasc Med 2009; 10: 852-8.

17. Urban P, Meredith I, Abizaid A, et al. Polymer-free drug-coated coronary stents in patients at high bleeding risk. N Engl J Med 2015; 373: 2038-47.

18. Windecker S, Kolh P, Alfonso F, et al. 2014 ESC/EACTS Guidelines on myocardial revascularization: the Task Force on Myocardial Revascularization of the European Society of Cardiology (ESC) and the European Association for Cardio-Thoracic Surgery (EACTS). Developed with the special contribution of the European Association of Percutaneous Cardiovascular Interventions (EAPCI). Eur Heart J 2014; 35: 2541-619.

19. Morice MC, Urban P, Greene S, et al. Why are we still using coronary bare-metal stents? J Am Coll Cardiol 2013; 61: 1122-3. 